Kluß, Nicola

\title{
Ernährungswissen und -handeln am Beispiel von Vollkorn. Ein
}

\section{Lehr-Forschungsprojekt}

Haushalt in Bildung \& Forschung 3 (2014) 3, S. 109-120

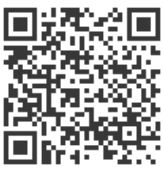

Quellenangabe/ Reference:

Kluß, Nicola: Ernährungswissen und -handeln am Beispiel von Vollkorn. Ein Lehr-Forschungsprojekt In: Haushalt in Bildung \& Forschung 3 (2014) 3, S. 109-120 - URN: urn:nbn:de:0111-pedocs-204298 DOI: 10.25656/01:20429

https://nbn-resolving.org/urn:nbn:de:0111-pedocs-204298

https://doi.org/10.25656/01:20429

in Kooperation mit / in cooperation with:

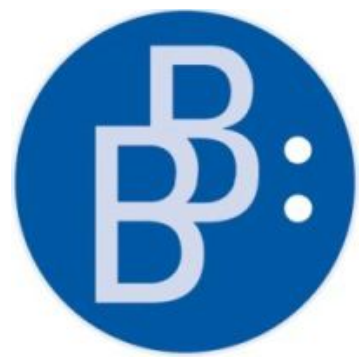

https://www.budrich.de

\section{Nutzungsbedingungen}

Gewährt wird ein nicht exklusives, nicht übertragbares, persönliches und beschränktes Recht auf Nutzung dieses Dokuments. Dieses Dokument is ausschließlich für den persönlichen, nicht-kommerziellen Gebrauch bestimmt. Die Nutzung stellt keine Übertragung des Eigentumsrechts an diesem Dokument dar und gilt vorbehaltlich der folgenden Einschränkungen: Auf sämtlichen Kopien dieses Dokuments müssen alle Urheberrechtshinweise und sonstigen Hinweise auf gesetzlichen Schutz beibehalten werden. Sie dürfen dieses Dokument nicht in irgendeiner Weise abändern, noch dürfen Sie dieses Dokument für öffentliche oder kommerzielle Zwecke vervielfältigen, öffentlich ausstellen, aufführen, vertreiben oder anderweitig nutzen.

Mit der Verwendung dieses Dokuments erkennen Sie die Nutzungsbedingungen an.

\section{Terms of use}

We grant a non-exclusive, non-transferable, individual and limited right to using this document.

This document is solely intended for your personal, non-commercial use. Use of this document does not include any transfer of property rights and it is conditional to the following limitations: All of the copies of this documents mus retain all copyright information and other information regarding legal protection. You are not allowed to alter this document in any way, to copy it for public or commercial purposes, to exhibit the document in public, to perform, distribute or otherwise use the document in public.

By using this particular document, you accept the above-stated conditions of use.

\section{Kontakt / Contact:}

\section{peDOCS}

DIPF | Leibniz-Institut für Bildungsforschung und Bildungsinformation Informationszentrum (IZ) Bildung

E-Mail: pedocs@dipf.de

Internet: www.pedocs.de

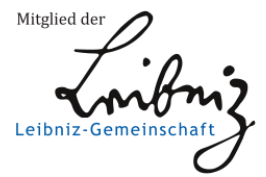


3. Jahrgang Heft 3

2014

는

๓)

$\bullet$

(1)

$\frac{1}{\bar{E}}$

0

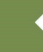

(1)

(

+

ᄃ

믕

은

논

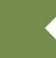

๕

(1)



$\varepsilon$

ह

$\frac{\mathcal{B}}{\mathrm{N}}$

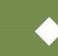

$+$

$\stackrel{\$}{ٍ}$

$\bullet$

$\frac{1}{\Phi}$
$\frac{c}{0}$
$\frac{5}{5}$
d
0

\section{Haushalt in Forschung}

\section{Subjektorientierung in der}

\section{Ernährungs- und Verbraucherbildung}


Inhaltsverzeichnis |

Silke Bartsch \& Claudia Wespi

Editorial. 2

Silke Bartsch \& Barbara Methfessel

Der ,subjektive Faktor“. Bildung in einem lebensweltorientierten Fach 3

Werner Brandl

Bausteine und Baustelle einer Didaktik subjektorientierten Lernens und Lehrens... 33

Claudia Wespi \& Corinne Senn Keller

Subjektorientiertes Lernen und Lehren in einer kompetenzorientierten

Unterrichtskonzeption

Käthi Theiler-Scherrer \& Corinne Senn Keller

Simulationsspiel als subjektorientierte Konsumbildung 75

Barbara Methfessel \& Bärbel Schön

Biographisch orientierte Lehr-Lernprozesse als „Brücke“ zwischen

lebensweltlichen Erfahrungen und systematischen Lehr-Lernprozessen -

Erfahrungen aus einem Lehr-Forschungsprojekt.

Nicola Kluß

Ernährungswissen und -handeln am Beispiel von Vollkorn - ein Lehr-

Forschungsprojekt.

Werner Brandl

Rezension: Didaktische Prinzipien.

Melanie Lukas

Rezension: Wesen und Wege nachhaltigen Konsums. 


\section{Nicola Kluß}

\section{Ernährungswissen und -handeln am Beispiel von Vollkorn. Ein Lehr-Forschungsprojekt}

Für die Professionalisierung von Lehramtsstudierenden der Ernährungs- und Verbraucherbildung (EVB) ist wichtig, dass Fachwissen auch für die Fachpraxis handlungsleitend wird. Im Lehr-Forschungsprojekt ${ }^{1}$ wird am Beispiel von Vollkornprodukten aufgezeigt, dass dieses durch entsprechende Lehr-Lernarrangements in der Hochschullehre angebahnt werden muss.

Schlüsselwörter: Ernährungswissen, Erfahrungswissen, Ernährungshandeln, Ernährungsund Verbraucherbildung

\section{Einleitung}

\subsection{Ausgangssituation}

Die Pädagogische Hochschule in Heidelberg bietet für Lehramtsstudierende des Faches „Alltagskultur und Gesundheit“" Seminare an, die die drei Arbeitsschwerpunkte des Faches, Fachwissenschaft, Fachdidaktik und Fachpraxis miteinander verbinden. Diese sollen die zukünftigen Lehrkräfte dazu befähigen, Jugendliche neben fundierten Fachkenntnissen und Fertigkeiten in der Fachpraxis für einen selbstverantwortlichen Lebens- und Essstil zu sensibilisieren. Bei der Zubereitung und Verkostung von Mehlspeisen in den fachpraktischen Seminaren fiel auf, dass die Studierenden fast ausnahmslos zu der Mehltype 405 griffen. Sie benannten diese Mehltype als das „,normale“ Mehl. Das hatte zur Folge, dass kaum Geschmackserfahrungen über Weißmehl hinaus im Seminar möglich waren. Diese Ausgangssituation forderte zur Veränderung heraus. Dabei stellt sich nachfolgende Frage: Wie kann erreicht werden, dass Studierende auch Mehle mit höherem Ausmahlungsgrad bei der Nahrungszubereitung berücksichtigen?

\subsection{Warum gerade Vollkorn?}

In Anbetracht der alarmierenden Zahlen zum Gewichtsstatus (z. B. Hölling et al., 2012) und zum Essverhalten von Jugendlichen (z. B. Max-Rubner-Institut, 2008) leitet sich als eine Aufgabe der Ernährungsbildung ab, das Ernährungswissen und das Spektrum an (gesundheitsförderlichen) Handlungsalternativen zu erweitern. Die Erfüllung dieser Aufgabe wird jedoch in Frage gestellt, wenn ernährungsphysiologisch wertvolle Lebensmittel wie Gemüse oder hier Vollkornprodukte bei der 


\section{Vom Wissen zum Handeln}

Nahrungszubereitung von Lehramtsstudierenden nicht von Anfang an genutzt werden (Bartsch et al., 2013). Im Sinne eines subjektorientierten Vorgehens sollten dazu Erfahrungswissen und -handeln der Schülerinnen und Schüler in den LehrLernprozessen gezielt aufgegriffen, analysiert und reflektiert werden (Bartsch, 2008; 2012; Brandl, 2008).

Im Lehr-Forschungsprojekt sollen am Beispiel von Vollkornprodukten Geschmacksbarrieren bei den Studierenden aufgespürt, hinterfragt und gegebenenfalls erweitert werden. Die Intention ist, angehende Lehrkräfte in die Lage zu versetzen, eine reflektierte Planung, Zubereitung und Verkostung von Speisen mit ihren Lerngruppen durchzuführen. Dazu sollen sie ihr Ernährungswissen erweitern und für die Genese ihrer eigenen Ernährungsgewohnheiten sensibilisiert werden.

Dies beinhaltet, sich mit Grundnahrungsmitteln während des Studiums hinsichtlich ihres Gesundheitswertes auseinanderzusetzen, unabhängig von (bisherigen) persönlichen Vorlieben. Ziel ist, Studierende zu befähigen, Lebensmittel mit einer hohen Nährstoffdichte wie Vollkornprodukte regelmäßig und selbstverständlich in der Schulpraxis einzusetzen. Ausgehend von der Seminarsituation und den DGE Ernährungsempfehlungen (DGE, 2013), wurden Vollkornprodukte als Beispiel gewählt. Diese gehören zu der Gruppe der nährstoffdichten Lebensmittel, die mit einer geringen Energiedichte einen hohen Gehalt an Vitaminen, Mineral- und Ballaststoffen aufweisen. Die Sättigung hält durch den hohen Ballaststoffgehalt verhältnismäßig lange an, die Insulinausschüttung ist gemäßigt. Darüber hinaus geht es im Seminar darum, die von der DGE (ebd.) und der Vollwerternährung (von Körber et al., 2012) empfohlene Norm zu diskutieren und zu reflektieren sowie eventuelle Verzehrbarrieren gegenüber den empfohlenen Lebensmitteln zu hinterfragen.

\section{Ernährungswissen und Erfahrungswissen}

Die befragten Studierenden bringen unterschiedliches Vorwissen aus Alltag und Schule mit. Allen gemeinsam ist, dass sie die Grundlagenvorlesung „Ernährung und Esskultur“ besucht haben. In dieser spielt „Vollkorn“ z. B. in Bezug auf Nährstoffe bzw. als Teil von Ernährungskonzepten eine Rolle. Die Fachinhalte wurden über eine Klausur abgeprüft.

Das in der Vorlesung thematisierte Fachwissen scheint jedoch weder für privates noch für zukünftig professionelles Handeln leitend zu sein, so die Beobachtung aus den Seminaren. Beispielsweise nutzten die Studierenden für ihre Nahrungszubereitung kaum Vollkornprodukte, ohne dazu explizit aufgefordert zu werden. $\mathrm{Zu}$ vermuten ist, dass das in Vorlesungen vermittelte Wissen mit den außerschulischen Kenntnissen und Vorerfahrungen der Studierenden verbunden werden muss, um zu ernährungsförderlichem Handeln im privaten wie professionellen Bereich zu führen (Bartsch, 2008; Bartsch \& Methfessel, i. d. H.). Mögliche Vorurteile und bisherige Erfahrungen der Studierenden sollen dazu aufgegriffen und deren Hintergrün- 


\section{Vom Wissen zum Handeln}

de für Ernährungsgewohnheiten beleuchtet sowie den Kenntnissen über Ernährung gegenübergestellt werden. Lehrkräfte in der Ernährungsbildung sollten reflektiert mit der eigenen Essbiographie umgehen können (vgl. Methfessel \& Schön, i. d. H.). Nur, wenn sie eigene Barrieren kennen, kann es gelingen, auch bei Lernenden Ernährungswissen mit Ernährungsgewohnheiten in Einklang zu bringen.

\section{Ziel der Studie}

In der vorliegenden Studie soll am Beispiel der Nutzung von Vollkornprodukten geklärt werden, welchen Wissens- und Handlungszuwachs Lehramtsstudierende aus den fachwissenschaftlichen und fachpraktischen Seminaren mitnehmen. Die Ergebnisse der Untersuchung sollen helfen, die Lehre der fachpraktisch ausgerichteten Seminare weiter zu entwickeln. Erste Ergebnisse liegen dazu vor (Kluß \& Bartsch, 2013). Aufgrund der gemachten Erfahrungen (ebd.) können folgende Hypothesen aufgestellt werden: Lehrerinnen und Lehrer können eine gesundheitsförderliche Essweise bei Kindern und Jugendlichen fördern, wenn sie

1. bei der Nahrungszubereitung sowie der Verkostung von Speisen eine Vielfalt gesundheitsförderlicher Lebensmittel verwenden (dazu gehören auch Vollkornmehle),

2. ihr eigenes, von den Ernährungsempfehlungen der DGE oder dem Ernährungskonzept der Vollwerternährung abweichendes Verhalten bewusst wahrnehmen und lernen, dieses auf der professionellen Ebene als Lehrende zu reflektieren.

\section{Methode}

An der Pädagogischen Hochschule in Heidelberg wurden im Lehr-Forschungsprojekt explorative Interventionsstudien durchgeführt: Zu Beginn (Prätest) und am Ende (Posttest) der entsprechenden Lehrveranstaltung wurde mittels Fragebogen untersucht, ob (1) die Intervention erfolgreich war, d. h. sich das Fachwissen vergrößert hatte und (2) inwiefern das Wissen um ernährungsförderliche Lebensmittel, hier am Beispiel von Vollkornprodukten, nun besser mit dem Alltags- bzw. dem professionellen Handeln von Lehramtsstudierenden verbunden werden konnte.

Da die erhofften Veränderungen auf Seiten der Studierenden durch verschiedene Interventionen im Laufe der Seminare bewirkt werden sollten, erfolgen zunächst einige Ausführungen zu diesen. In zwei Seminarsitzungen wurden zunächst fachwissenschaftlich die Unterschiede zwischen Auszugsmehlen und Vollkornmehlen sowie ihrer Auswirkungen auf den Körper vorgestellt und diskutiert. Darüber hinaus wurden die Zubereitungspraxis und die Verkostung im Hinblick auf die Verwendung unterschiedlicher Mehltypen gemeinsam mit den Studierenden geplant, durchgeführt 


\section{Vom Wissen zum Handeln}

und ausgewertet. Damit wurde sichergestellt, dass alle Speisen aus Auszugsmehlen, Mischmehlen und Vollkornmehlen hergestellt wurden. Die Speisen wurden dann verkostet, Unterschiede und Gemeinsamkeiten in Aussehen, Geruch, Geschmack und Konsistenz gegenüber gestellt und bewertet. Dies erfolgte bei jeder der zehn Sitzungen im Plenum.

Ziel der Intervention war, dass Studierende ihr eigenes Essverhalten bezüglich Vollkornprodukten reflektieren und möglicherweise ihr Essverhalten ändern. In den Lehrveranstaltungen wurde z. B. über Geschmacksbarrieren diskutiert, Befindlichkeiten bei neuen Geschmackserlebnissen beschrieben und bewertet. Auf eine offene und wertschätzende Atmosphäre zwischen der Dozentin und den Lernenden wurde Wert gelegt, um die Gefahr von sozial erwarteten Antworten in den Befragungen zu reduzieren. Die Studierenden stellten während der gesamten Seminardauer häufig Verständnisfragen zu Vollkornprodukten und während der Nahrungszubereitung zu deren küchentechnischen Eigenschaften. Diese wurden umfassend beantwortet, auch wenn es sich zum Teil um Fragen handelte, die die Studierenden aufgrund ihres vorangegangenen Studiums schon hätten wissen müssen. Während der Verkostung wurden die individuellen Vorstellungen der Studierenden, wie die zubereiteten Speisen ihrer Meinung nach schmecken sollten, mit dem Geschmack der tatsächlich zubereiteten Speisen durch sensorische Beschreibungen verglichen. Es gab keine Beoder Abwertung von vorgenommenen Bewertungen oder Haltungen gegenüber den selbst hergestellten Produkten. Darüber sollten eigene Geschmacksvorlieben reflektiert und gegebenenfalls erweitert werden.

Insgesamt 72 Lehramtsstudierende des Faches „Alltagskultur und Gesundheit“, die später in der Sekundarstufe I unterrichten wollen, nahmen an der Befragung teil. Typisch für die Domäne EVB war die geringe Zahl der teilnehmenden männlichen Befragten mit nur $4 \%(N=3)$ (Bartsch \& Methfessel, 2012). Das Alter der meisten Studierenden lag zwischen 21 bis 25 Jahren, nur zwei Teilnehmerinnen waren zwischen 33 bis 50 Jahren alt. Fast $70 \%$ der Befragten hatten ein Gymnasium besucht: Mehr als Zweidrittel der Studierenden hatten somit das Fach, das sie später lehren sollen, als Schülerin und Schüler selbst nicht kennen gelernt und keine eigenen Schulesserfahrungen gesammelt.

Der Fragebogen enthält überwiegend geschlossene Fragen zum (1) Ernährungswissen, (2) dem Konsum von Vollkornprodukten, (3) zur Ernährungssozialisation und (4) zu den eigenen Schulesserfahrungen der Studierenden. Die Antwortmöglichkeiten waren entweder dichotom als Ja-Nein (1-0) oder in Form von Ratingskalen mit vier bis fünf Stufen von vollkommen zutreffend (1) bis überhaupt nicht zutreffend $(4 ; 5)$ formuliert. Der Fragebogen umfasst folgende Teile:

(1) Ernährungswissen

Mit Fragen nach den Inhaltsstoffen von Vollkornprodukten z. B. bezüglich des höheren Vitamin-, Mineralstoff- und Ballaststoffgehalts, sollte das Wissen der Studierenden über die Nährstoffverteilung bei Vollkornprodukten differenziert erfasst werden. 


\section{Vom Wissen zum Handeln}

(2) Konsum von Vollkornprodukten

Diese Fragen beziehen sich auf den Essalltag der Studierenden, beispielsweise wie oft sie Vollkornmehl, Mischmehl bzw. Auszugsmehl bei der privaten Nahrungszubereitung verwenden und welche Vollkornprodukte sie wie häufig kaufen.

(3) Ernährungssozialisation

Mit diesen Fragen soll erfasst werden, ob in der Herkunftsfamilie der Studierenden Vollkornmehle verwendet bzw. welche Produkte wie oft als Vollkornvariante gekauft wurden.

(4) Eigene Schulesserfahrungen

Hier wird gefragt, welche Erfahrungen die Studierenden mit Vollkornprodukten in der Schule und während des Studiums im Umgang mit Ausbilderinnen/Ausbildern gemacht haben. Zudem sollen sie aufschreiben, welche Erwartungen sie an sich von Seiten der Schule gerichtet sehen, Vollkornprodukte mit einzubeziehen.

Im Sommersemester 2012 wurde der Fragebogen erstmals eingesetzt und aufgrund der Erfahrungen optimiert. Die standardisierte Befragung fand zu Beginn und am Ende von zwei zehnwöchigen Seminaren (,Kultur und Technik der Nahrungszubereitung“ und „Ausgewählte Fragestellungen zur Nahrungs-, Mahlzeiten- und Esskultur") im Wintersemester 2012/13 und im Wintersemester 2013/2014 statt (vgl. dazu Kluß \& Bartsch, 2013). Die Teilnahme war freiwillig und anonym. Die Teilnehmenden trugen einen persönlichen Code in die Kopfzeile des Fragebogens ein, so dass Eingangs- und Ausgangsbefragungsbogen individuell zugeordnet werden können. Die Daten wurden mit den entsprechenden Statistikroutinen des Statistikprogrammpaketes SPSS ausgewertet.

\section{Ergebnisse}

Aufgrund der Datenfülle werden nur Ergebnisauszüge, vorrangig zur Beantwortung der in diesem Beitrag aufgeworfenen Fragestellungen (siehe 1.1), dargestellt. Die Darstellung beschränkt sich dabei im Wesentlichen auf Ergebnisse, bei denen statistische Signifikanz (Irrtumsrisiko $\alpha<0.05$ ) festgestellt wurde.

\subsection{Fachwissen}

Die hier zu beantwortende Frage war, ob das Fachwissen der Studierenden nach der Intervention, d. h. am Ende der Lehrveranstaltung, im Sinne des Lehrzieles angestiegen ist. Die zweifache Varianzanalyse mit den beiden Hauptfaktoren Studienjahrgang (WS 2012/13 und WS 2013/14) und dem Studienjahr (Modul 2 und Modul 3) sowie dem Messwiederholungsfaktor (Veranstaltungsbeginn und -ende) bestätigt den Wissenszuwachs: Zum einen ergibt sich ein Effekt der Lehre 


\section{Vom Wissen zum Handeln}

$(F=15.80 ; p<0.001)$, zum anderen aber auch ein Effekt der Studienjahrgänge $(F=5.91 ; p=0.02)$ : Der zweite Studienjahrgang startet auf einem höheren Niveau des Fachwissens als der erste Studienjahrgang. Ein erwartbarer Effekt des Studienjahres (Modul 2 vs. Modul 3) bleibt hingegen aus, d. h. die Studierenden im Modul 3 , die einen Wissensvorsprung gegenüber den Studierenden im Modul 2 haben sollten, erreichen keine besseren Werte. Zwar steigt das Fachwissen bezüglich Vollkornprodukten durch die Lehre an, dennoch ist erstaunlich, dass zwischen den Studienjahren kein Wissenszuwachs zu erkennen ist.

\subsection{Geschmack, Aussehen und Verzehrhäufigkeit}

Nach Pudel und Westenhöfer (2003) beeinflussen (pseudo-)rationale Einstellungen das Ernährungsverhalten, und zwar in Abhängigkeit vom Lebensalter. Ältere Personen lassen sich mehr davon leiten als jüngere. Bei Jüngeren sind eher die Außenreize handlungsleitend. Aus diesem Grund interessierte: Sind im Lebensalter der Studierenden schon die rationalen Einstellungen zum Ernährungswissen handlungsleitend, so dass sie durch mehr Wissen auch mehr Vollkornprodukte konsumieren? Zur Beantwortung wurden vor allem die Antworten auf die Fragen herangezogen, die Aufschluss über das Konsumieren von Vollkornprodukten durch deren Kauf oder Herstellung geben. Zunächst wird im Folgenden dargestellt, inwiefern sich die Bewertungen hinsichtlich Aussehen und Geschmack von Vollkornprodukten durch die Intervention bei den Studierenden ändern.

Die Beurteilung des Geschmacks von Vollkornprodukten verändert sich durch die Lehre statistisch nicht signifikant, die Geschmackspräferenzen sind in Prä- und Posttest vergleichbar, wie dies die Skalenmittel zu Beginn und am Ende der Veranstaltung zeigen $\left(M_{1}=1.8 ; S D_{1}=0.6\right.$ vs. $M_{2}=1.7 ; S D_{2}=0.5 ; t_{\text {(abhängig) }}=0.85$; $p=0.40)$. Die Korrelation ist hingegen statistisch bedeutsam, wenn auch nicht besonders eng $(r=.38 ; p<0.01)$.

Vergleichbare Ergebnisse wie beim Geschmack zeigen sich bei der Bewertung des Aussehens von Vollkornprodukten: Zwischen Beginn und Ende des Seminares verändern sich die Beurteilungen statistisch nicht bedeutsam $\left(M_{l}=2.4 ; S D_{l}=1.4\right.$; $M_{2}=2.2 ; S D_{2}=1.4 ; t_{\text {(abhängig) }}=0.96 ; p=0.34$ ). Ein statistisch bedeutsamer Zusammenhang besteht aber wieder zwischen den beiden Beurteilungen $(r=0.38$; $p<0.01)$.

Die Studierenden bewerten am Anfang des Semesters das Aussehen von Vollkornprodukten mehrheitlich als „ansprechend“. Am Ende des Semesters ist den meisten Studierenden das Aussehen ,egal“. Diese Veränderung in der Antwortverteilung ist statistisch signifikant (exakter Test nach Fisher $25.4(p<0.001)$.

Die Studierenden beider Studienjahrgänge kreuzen am Ende des Seminars bedeutsam mehr Vollkornprodukte (Nudeln, Reis, Brot, Pfannkuchen, Gebäck und andere) an, die sie essen, als sie dies zu Beginn angegeben haben $\left(t_{\text {(abhängig) }}=7.02\right.$; $p<0.001)$. Die zweifache Varianzanalyse mit den Faktoren Studienjahrgang 
(WS 2012/13 und WS 2013/14) und Studienjahr (Modul 2 und Modul 3) sowie dem Wiederholungsfaktor „Lehre“ (Beginn und Ende) zeigt allerdings ein differenzierteres Bild. Der Studienjahrgang wirkt sich auf die Zahl gerne gegessener Vollkornprodukte insofern aus, als die Studierenden des Jahrgangs 2013/14 zwar vergleichbar viele Vollkornprodukte essen wie die des Jahrgangs 2012/13. Während aber beim Jahrgang 2012/13 ein bedeutsamer Effekt durch die Lehre nachgewiesen werden kann, ist dies beim Jahrgang 2013/14 nicht der Fall.

Die Modul 3-Studierenden konsumieren zu Beginn der Lehrveranstaltung mehr Vollkornprodukte als die Modul 2-Studierenden. Im Durchschnitt steigt in beiden Modulen zwischen Beginn und Ende der Lehrveranstaltung der Verzehr von Vollkornprodukten um insgesamt 1,2 der anzukreuzenden Produkte (Nudeln, Reis, Brot, Pfannkuchen, Gebäck und andere) an. Hat z. B. zu Beginn der Lehrveranstaltung ein Studierender des Modul 2b Seminars im Durchschnitt 2,5 der anzukreuzenden Produkte als Vollkornvariante verzehrt, so sind dies am Ende der Veranstaltung durchschnittlich 3,7 der verschiedenen Produkte.

Aufgrund zum Teil fehlerhafter Kodierungen der Eingangs- und Ausgangsfragebögen der Studierenden war die Frage leider nicht zu beantworten, ob ein erhöhtes Fachwissen mit einem erhöhten Konsum von Vollkornprodukten in Beziehung steht.

\subsection{Geplanter Einsatz von Vollkornprodukten in der Schule}

Rund $90 \%$ der Befragten gaben an, dass sie als zukünftige Lehrerin oder Lehrer regelmäßig Vollkornprodukte in der Schule einsetzen möchten. Hiervon würden ca. $55 \%$ Vollkornprodukte jedes Mal im Unterricht berücksichtigen, rund $43 \%$ alle paar Wochen.

Die Frage nach den Erwartungen, die die Schule u. a. von Seiten des Kollegiums, des Rektors, der Eltern an die künftigen Lehrkräfte richtet, war offen zu beantworten. Nahezu alle Studierende gaben an, dass sie davon ausgehen, Vollkornprodukte im Rahmen einer vollwertigen Ernährung in der Schule anbieten zu müssen. Etwa ein Drittel der Studierenden notierte zudem, dass nur durch den regelmäßigen Einsatz von Vollkornprodukten eine Geschmackserweiterung bei den Kindern und Jugendlichen erzielt werden kann.

\subsection{Esserlebnisse}

Bei dieser offen formulierten Frage sollten die Studierenden angeben, ob sie sich an positive oder negative Esserlebnisse beim Verzehr von Vollkornprodukten erinnern konnten. Nur wenige beantworteten diese Frage. Eindeutig war jedoch, dass positive Erfahrungen stets mit einer positiven Essatmosphäre verbunden wurden (z. B. Geburtstage, besonderes Erlebnis beim Bäcker oder bei der Oma). Das entspricht dem Stand der Literatur (Bartsch, Methfessel \& Schlegel-Matthies, 2006). Negative Esserlebnisse beruhten zumeist auf einer Situation, die als erdrückend 


\section{Vom Wissen zum Handeln}

oder als Zwang empfunden wurden, wie z. B. nachfolgende Erlebnisse von Studierenden zeigen: „Beim Kindergeburtstag eines Freundes gab es Vollkornspaghetti mit Ketchup. Nachdem ich das nicht gegessen und die Mutter des Geburtstagskindes meiner Mutter davon berichtet hatte, bekam ich Ärger “. „In der Prüfungswoche wollte ich meine Lieblingsspeise, Kaiserschmarrn, zubereiten, hatte aber nur Vollkornmehl da. Ich dachte, ich beiße in etwas Leckeres aus meiner Kindheit, aber dem war nicht so. Ich war sehr enttäuscht und das zog mich runter. “

\section{Diskussion}

\subsection{Fachwissen}

Die Lehre hat den gewünschten Effekt erzielt. Ein Zuwachs von Wissen ist bei den Studierenden zu verzeichnen. Dennoch bleibt zu hinterfragen, warum Modul 3 Studierende gegenüber Modul 2 Studierenden keinen Wissensvorsprung aufweisen. Hier kann vermutet werden, dass das vermittelte Fachwissen zu den bisherigen Erfahrungen und Einstellungen der Modul 3 Studierenden im Widerspruch steht, so dass mittel- und langfristig auf gewohntes Ernährungsverhalten zurückgegriffen wird. Es kann aber auch gezeigt werden, dass ein Zusammenhang zwischen rationalen Einstellungen und dem Ernährungsverhalten bei den Studierenden besteht (vgl. Pudel \& Westenhöfer, 2003). So schreibt eine Studentin: „Ich ernähre mich viel öfter von Vollkornprodukten, seitdem ich weiß, dass sie viel gesünder sind" (S. 47).

In Modul 2 geht es um Fachwissen, das sowohl für die Nahrungszubereitung bedeutsam ist als auch bei der Verkostung thematisiert wird. Bezüglich Vollkorn bedeutet dies, dass die Studierenden in der Theorie die Inhaltsstoffe dieser Produkte kennen lernen und in Bezug zu Auszugsmehlen setzen. In der Praxis erleben die Studierenden die küchentechnischen Eigenschaften verschiedener Mehltypen. Während der Verkostung beschreiben die Studierenden zunächst Geschmack, Aussehen, Konsistenz, Geruch, um daran anschließend einen Bezug zu ihren Vorlieben herzustellen. Bevor die Lernenden das Seminar Modul 3 besuchen, vergehen in der Regel ein bis zwei Semester. In dieser Zeit manifestiert sich das Erlernte oder verliert sich wieder. Für die Lehre bedeutet dies, dass noch stärker gelebter Alltag mit den dahinterstehenden Vor- und Einstellungen der Studierenden im Hochschulseminar thematisiert und reflektiert sowie mit Wissenschaftstheorien verknüpft werden muss (vgl. Bartsch \& Methfessel, i. d. H). Dauerhaft muss in den betreffenden Seminaren die Aufarbeitung und Reflexion der Einstellungen und Vorurteile der Studierenden (z. B. „,Vollkorn kann ich mir nicht leisten “; ,Vollkorn ist grob und passt nicht zu Süßspeisen “; „Vollkorn ist schwer zu verarbeiten") und die Verknüpfung mit Fachwissen und Fachpraxis erfolgen. 


\section{Vom Wissen zum Handeln}

\subsection{Aussehen, Geschmack und Verzehrhäufigkeit}

Das dunklere Aussehen von Vollkornprodukten scheint den Ergebnissen nach kein Grund zu sein, Vollkornprodukte abzulehnen. Dass das Aussehen der Vollkornprodukte durch die Studierenden am Ende der Veranstaltung mit „egal“ bewertet wird, zeigt außerdem, dass es im Verlauf des Semesters unwichtiger geworden ist.

Durch die zehnwöchige Lehre ließen sich die Geschmackspräferenzen der Studierenden nicht verändern. Wenig verwunderlich, so ist Geschmack Gewohnheit (Methfessel, 2005) - oder wie Bourdieu (1993) es formuliert - Teil des Habitus. Geschmack gibt Sicherheit; er verortet das Individuum in seinem Umfeld, grenzt ab und aus (Barlösius, 2011; Methfessel, 2005). Studienzeit ist eine Umbruchphase im Leben der Studierenden, in der sie nach neuen Erfahrungen streben und diese häufig aufgrund des Auszuges aus dem Elternhaus auch machen. Situationen mit biografischen Übergängen bergen die Chance, Bisheriges zu überdenken, Neues zu erproben und (veränderte) Alltagsroutinen beizubehalten oder zu etablieren (Brombach, 2000).

\subsection{Esserlebnisse - Verzehr von Vollkornprodukten}

Positive und negative Esserlebnisse sind für den weiteren Umgang mit den jeweiligen Lebensmitteln und Speisen überwiegend prägend (vgl. Methfessel, 2005 und die dort angegebene Literatur). Um Jugendlichen sowie Studierenden positive Esserfahrungen mit Vollkornprodukten zu ermöglichen und darüber den langfristigen Konsum zu erhöhen, bedarf es einer offenen, wertschätzenden Lernumgebung, die ohne Zwang und Druck Erfahrungen im Umgang mit neuen Speisen zulässt. Bestehende Geschmäcker dürfen dabei nicht diffamiert, abgewertet oder ausgegrenzt werden (vgl. dazu auch Bartsch, Methfessel \& Schlegel-Matthies, 2006).

Die Zahl konsumierter Vollkornprodukte hat sich bei den Studierenden nach der zehnwöchigen Lehre erhöht. Dies spricht zum einen dafür, dass im Seminar durch den wertschätzenden Umgang miteinander neue positive Geschmackserlebnisse ermöglicht wurden. Zum anderen konnten die Studierenden durch die Lehre und das damit einhergehende vielfältigere Angebot an Vollkornprodukten neue Alternativen für den eigenen Verzehr erleben. Wenn auch, wie oben beschrieben, die Geschmacksvorlieben verhältnismäßig stabil sind, konsumieren die Modul 3 Studierenden mehr Vollkornprodukte als die Modul 2 Studierenden. Vorsichtig kann dies als Indiz gewertet werden, dass das curriculare Angebot von zwei aufeinander aufbauenden Lehrveranstaltungen zu einer Erweiterung bzw. Ergänzung des Verzehrs von Vollkornprodukten beigetragen hat.

Beim Vergleich der Studienjahre sind die Unterschiede bezüglich des Verzehrs von Vollkornprodukten nur gering. Dies könnte ein Indiz dafür sein, dass sich nicht nur der Geschmack, sondern auch Gewohnheiten wie der Einkauf von Lebensmitteln schwierig und zeitaufwändig verändern lassen. Das Vorhaben, Vollkornprodukte zu kaufen, könnte u. a. schon daran scheitern, dass keine, dem eigenen Geschmack und 


\section{Vom Wissen zum Handeln}

dem eigenen Geldbeutel passenden Geschäfte auf dem alltäglich zurückgelegten Arbeitsweg liegen.

Damit im Rahmen der Hochschule vermitteltes Ernährungswissen mit dem eigenen Ernährungshandeln verknüpft werden kann, müssen über konkrete Alltagssituationen Handlungsalternativen angeboten werden. Im Allgemeinen lassen sich neue Handlungsmuster im Essalltag gut integrieren, wenn sie in den persönlichen Alltagsablauf ohne große Schwierigkeiten einzubauen sind. Solch eine Alltagssituation ist $\mathrm{u}$. a. der Einkauf von Lebensmitteln, der Teil der Fachpraxis ist (Bartsch \& Bürkle, 2013). In den Seminaren stellten die Studierenden häufig die Frage, wo es auf ihrem Weg zur Hochschule oder nach Hause preiswert Vollkornprodukte zu kaufen gäbe.

\subsection{Einsatz von Vollkornprodukten in der Schule}

Aus hochschuldidaktischer Sicht ist insbesondere die Lehrkompetenz zur Vernetzung von „Theorie und Praxis“ in Unterrichtssituationen mit Nahrungszubereitung interessant (Bartsch \& Bürkle, 2013). Die Antworten der Studierenden zeigen, dass alle davon ausgehen, Vollkornprodukte in der Schule einsetzen zu müssen. Nahezu alle Studierende gaben zudem an, Vollkornprodukte in der Schule einsetzen zu wollen. Rund die Hälfte beabsichtigt dies bei jeder Nahrungszubereitung bzw. Verkostung, was aber gleichsam bedeutet, dass die andere Hälfte der Studierenden Vollkorn nur alle paar Wochen einsetzen würde. Verkannt wird aber m. E. wie bedeutsam es ist, Vollkorn neben anderen Mehlen immer als Teil einer vollwertigen Ernährung anzubieten. Es sollte nicht als das „,andere Mehl“, das „,dunkle Mehl“, das „,besondere Mehl“" von den Schülerinnen und Schülern gesehen werden, sondern immer als eine „normale“ Möglichkeit, Speisen zuzubereiten. Werden im Schulalltag Begriffe wie das ,normale Mehl“ von den Lernenden verwendet, gilt es über die dahinter stehende Bedeutung zu reflektieren, denn Sprache schafft Wirklichkeit. Wenn bei der Zubereitung von Mehlspeisen völlig selbstverständlich auf verschiedene Mehltypen zurückgegriffen werden kann und wird, wird vorbildhaft und implizit immer der Nutzen und die Nutzung einer Vielfalt von Nahrungsmitteln präsentiert.

\section{Fazit}

Ziel der Verkostung von Speisen nach der gemeinsamen Nahrungszubereitung in den fachdidaktischen Seminaren mit Fachpraxis ist, die Speisen entsprechend der jeweiligen Zielsetzung, z. B. in Bezug auf Zusammensetzung, Aussehen, Geruch, Geschmack, Konsistenz etc. oder hinsichtlich positiver und negativer Voreinstellungen der Probierenden zu reflektieren und darüber eine Geschmackserweiterung anzubahnen (Bartsch \& Bürkle, 2013; Methfessel \& Schlegel-Matthies, 2013). Mit dem beschriebenen Lehr-Forschungsprojekt liegen Hinweise vor, dass dies sowohl bei Modul 2- als auch bei Modul 3-Studierenden gelungen ist. Die Seminarkonzep- 


\section{Vom Wissen zum Handeln}

tion sieht eine Verknüpfung der Vermittlung von Fachwissen, der Nahrungszubereitung sowie der Reflexion der zubereiteten Speisen vor, um eigenes Ernährungsverhalten zu hinterfragen, Geschmackserweiterungen zu fördern, und Handlungsalternativen aufzubauen. Bestätigen lässt sich, dass sich Geschmack als sehr stabil und nur längerfristig veränderbar erweist, trotz zunehmendem Fachwissen. Wenn sich Studierende an einschneidende Esserlebnisse mit Vollkornprodukten erinnern konnten, polarisierten diese meistens. Dies führte entweder zum bevorzugten Konsum dieser Lebensmittel oder Speisen oder zur völligen Ablehnung. Es geht somit auch in der Hochschullehre darum, positive Esserlebnisse zu ermöglichen.

Individuelle Erfahrungen, die den Alltag stark bestimmen und die bei Routinetätigkeiten und -entscheidungen handlungsleitend sind, werden im Seminar aufgegriffen, um sie den Studierenden bewusst zu machen. Die Reflexion von essbiografisch bedeutsamen Erlebnissen scheint ein Schlüssel für Modifikationen und Erweiterungen der bisherigen Handlungsroutinen und des Wissens zu sein (vgl. auch Methfessel \& Schön, i. d. H.). Auch bei der Zubereitung der Speisen sowie deren Verkostung erscheint es wichtig, an die individuellen Erfahrungen und Einstellungen anzuknüpfen. Eine positive Lehr-Lernatmosphäre ist dabei förderlich.

\section{Anmerkungen}

1 Herzlichen Dank an Prof. Dr. B. Methfessel und Prof. Dr. S. Bartsch für ihre Unterstützung bei der Durchführung der Untersuchung sowie Prof. Dr. B. Methfessel und Prof. Dr. H. Schöler bei der Auswertung und der Diskussion der Ergebnisse.

\section{Literatur}

Barlösius, E. (2011). Soziologie des Essens (2. Aufl.). Weinheim: Juventa Verlag. Bartsch, S., Methfessel, B. \& Schlegel-Matthies, K. (2006). Pizza, Pasta, Döner Kebab - Mittelmeerkost im Alltag deutscher Jugendlicher. Haushalt \& Bildung, 83(4), 17-26.

Bartsch, S. (2008). Subjektorientierung in der Ernährungs- und Gesundheitsbildung. Ernährung, 3, 100-106.

Bartsch, S. \& Methfessel, B. (2012). Haushaltslehre - Vom Emanzipations- zum Kompetenzdiskurs. In C. Wiepcke \& M. Kampshoff (Hrsg.), Handbuch Geschlechterforschung und Fachdidaktik (S. 199-212). Wiesbaden: VS Verlag für Sozialwissenschaften.

Bartsch, S. \& Bürkle, P. (2013). Lernort Küche. Nahrungszubereitung als methodischer Zugang zur Fachpraxis Ernährung. Haushalt in Bildung \& Forschung, 2(4), 18-31.

Kluß, N. \& Bartsch, S. (2013). Ernährungswissen und -einstellungen von Studierenden im Hinblick auf die Ausbildung von Professionalität. In DGE (Hrsg.), 


\section{Vom Wissen zum Handeln}

Proceedings of the German Nutrition Society. Abstractband zum 50. Wissenschaftlichen Kongress (Vol. 18, S. 77).

Bartsch, S. et al. (2013). Ernährungsbildung - Standort und Perspektiven. Ernährungs Umschau, 2, 84-95.

Bourdieu, P. (1993). Die feinen Unterschiede (6. Aufl.). Frankfurt am Main: Suhrkamp Taschenbuch Verlag.

Brandl, W. (2008). Unterricht/en in der Ernährungs- und Verbraucherbildung: Vom didaktischen Modell zur methodischen Modellierung. Haushalt \& Bildung, 85(3), 3-20.

Brombach, C. (2000). Ernährungsverhalten im Lebensverlauf von Frauen über 65 Jahren. Eine qualitativ biographische Untersuchung. Gießen: Köhler.

DGE (Hrsg.). (2013). Vollwertig essen und trinken nach den 10 Regeln der DGE. [www.dge.de/pdf/10-Regeln-der-DGE.pdf].

Hölling, H., Schlack, R., Kamtsiuris, P., Butschalowsky, H., Schlaud, M. \& Kurth, B. M. (2012). Die KIGGS-Studie. Bundesgesundheitsblatt - Gesundheitsforschung-Gesundheitsschutz, 6-7, 836-842.

Körber, K.W. v. et al. (2012). Vollwerternährung: Konzeption einer zeitgemäßen und nachhaltigen Ernährung (11. Aufl.). Heidelberg: Karl F. Haug Verlag.

Max-Rubner-Institut (Hrsg.). (2008). Nationale Verzehrsstudie II.

[www.mri.bund.de/fileadmin/Institute/EV/NVSII_Abschlussbericht_Teil_2.pdf

Methfessel, B. (2005). Fachwissenschaftliche Konzeption: Soziokulturelle Grundlagen der Ernährungsbildung. Paderborner Schriften zur Ernährungs- und Verbraucherbildung (Band 07/2005).

Methfessel, B. \& Schlegel-Matthies, K. (2013). Für eine veränderte Fachpraxis zur Kultur und Technik der Nahrungszubereitung und Mahlzeitengestaltung. Haushalt in Bildung \& Forschung, 2(4), 49-60.

Pudel, V. \& Westenhöfer, J. (2003). Ernährungspsychologie. Eine Einführung (3. Aufl.). Göttingen, Bern, Toronto, Seattle: Hogrefe Verlag.

\section{Verfasserin}

Dipl. Päd. ${ }^{\text {in }}$ Nicola Kluß

Pädagogische Hochschule Heidelberg

Im Neuenheimer Feld 561

D-69120 Heidelberg

E-Mail: kluss@ph-heidelberg.de

Internet: www.ph-heidelberg.de 